Cite this: Chem. Commun., 2014 50,8335

Received 8th April 2014

Accepted 14th May 2014

DOI: $10.1039 / c 4 c c 02580 f$

www.rsc.org/chemcomm

\section{The morphology transformation from helical nanofiber to helical nanotube in a diarylethene self-assembly system $\dagger$}

\author{
Yulong Duan, ${ }^{a}$ Shihai Yan, ${ }^{b}$ Xinhong Zhou, ${ }^{* c}$ Wei $X u,{ }^{d}$ Hongxia $X u^{a}{ }^{a}$ Zhihong Liu, ${ }^{a}$ \\ Lixue Zhang, ${ }^{a}$ Chuanjian Zhang, ${ }^{a}$ Guanglei Cui*a and Lishan Yao*a
}

A helical nanostructure can be obtained by self-assembly of a diarylethene derivative that bears two malononitrile substitutes in a tetrahydrofuran/water medium. It is revealed that the helical nanostructure changed from helical nanofiber to helical nanotube when the diarylethene monomer changed from the open-ring isomer to the closed-ring isomer upon irradiation with $365 \mathrm{~nm}$ ultraviolet light, meanwhile, the helix angle of the nanostructure changed from $50^{\circ} \pm$ $5^{\circ}$ to $75^{\circ} \pm 5^{\circ}$. There is a great possibility that the helical nanofibers and helical nanotubes are assembled from dimers as base units based on theoretical calculation and experimental results.

Helical structures are common structural motifs in biomolecules and play a pivotal role in structural stability, signal transduction, genetic reading out and transcription which are involved in the storage of genetic information in biological systems such as proteins and DNA. ${ }^{1}$ The artificial construction of a helical structure is significant to fabricate functional materials ${ }^{2}$ and understand the non-covalent forces that hold the helical structures together. ${ }^{3}$ The creation of artificial helical structures in relation to controlled self-assembly has received great interest in the past decades. ${ }^{4-7}$ However, the structure features of the helix have received relatively little attention and the architecture control of the helical structures remains a great challenge. ${ }^{8}$

As an optically switchable molecule, diarylethene has widespread applications in many fields such as molecular electronics, ${ }^{9}$

\footnotetext{
${ }^{a}$ Qingdao Key Lab of Solar Energy Utilization and Energy Storage Technology, Qingdao Institute of Bioenergy and Bioprocess Technology, Chinese Academy of Sciences, Qingdao, 266101, China. E-mail: cuigl@qibebt.ac.cn, yaols@qibebt.ac.cn; Fax: +86-0532-80662746

${ }^{b}$ Qingdao Agricultural University, Qingdao, 266101, China

${ }^{c}$ College of Chemistry and Molecular Engineering, Qingdao University of Science and Technology, Qingdao, 266101, China

${ }^{d}$ Institute of Chemistry, Chinese Academy of Sciences, Beijing 10019, China

$\dagger$ Electronic supplementary information (ESI) available: Materials; UV-Vis in THF solution; self-assembly methods; instruments and test methods; AFM and TEM images of the helical nanofibers and helical nanotubes; molecular electrostatic potential (ESP) contour maps; relative energy $\left(E_{\text {relative }}\right)$ and binding energy ( $\left.E_{\text {binding }}\right)$ of the dimers; images of the dimers employed for the theoretical calculation. See DOI: 10.1039/c4cc02580f
}

molecular magnetism, ${ }^{10}$ cell biology ${ }^{11}$ and surface wettability ${ }^{12}$ but there are very few studies about the helical self-assembly of diarylethene molecules as far as we know. ${ }^{13}$ Because the cyclization reaction of the diarylethene can generate a new chiral center, photochromic ring closure will produce a pair of enantiomers if the molecule has no chiral center. ${ }^{14}$ The optical switching between different chiral aggregated states or the optical switching between molecular chirality and supramolecular chirality may offer new prospects for the development of molecular memory materials or smart functional materials. So far as we know it is the first report of the construction of a helical nanofiber and a helical nanotube by self-assembly from the same diarylethene molecule.

The molecule presented here is based on a diarylethene photochromic unit functionalized with two malononitrile groups (Scheme S1, ESI $\dagger$ ); the synthesis of the compound was initially reported by Lehn et al. ${ }^{15}$ The ultraviolet-visible absorption spectra of both isomers are shown in Fig. S1 (ESI $\dagger$ ). In a tetrahydrofuran (THF) solution, irradiation with $365 \mathrm{~nm}$ ultraviolet light in a few minutes resulted in photo-cyclization to a photostationary state (PSS), meanwhile, the colour of the solution changed to cyan from colourless as a stiffer planar closed-ring isomer was generated. Subsequent irradiation with visible light led exclusively to the open-ring isomer and the colour faded to the initial state.

The self-assembly was performed in a THF-water mixture solution. The SEM characterization was performed on a mica chip. Fig. 1 shows that in this mixture solution helical nanofibers were formed by self-assembly. For the open-ring isomer, when the two solutions spread towards each other slowly at $30{ }^{\circ} \mathrm{C}$, nanofibers $>20 \mu \mathrm{m}$ long with obvious helical features were obtained. When the temperature was raised to $40{ }^{\circ} \mathrm{C}$, the helical nanofibers underwent slight aggregation. Moreover, it is demonstrated that flocs were generated from the helical nanofiber aggregates as the temperature was raised to $50{ }^{\circ} \mathrm{C}$. We think this may be because raising the temperature makes the molecules move faster, increasing the rate of aggregation. In the flocs, the diameters of the nanofibers ranged from tens to hundreds of nanometers and the fine nanofibers were wound 




Fig. 1 Typical SEM images and enlarged images of the helical nanofibers formed from the open-ring isomer of the diarylethene at different temperatures: (a, b) $30{ }^{\circ} \mathrm{C},(\mathrm{c}, \mathrm{d}) 40{ }^{\circ} \mathrm{C},(\mathrm{e}, \mathrm{f}) 50{ }^{\circ} \mathrm{C}$.

around the crude helical nanofibers, which coiled into superhelix structures; the secondary self-assembly properties have been reported previously. ${ }^{13,16}$

Temperature-dependent UV-Vis studies were performed in order to investigate the self-assembling properties of the diarylethene derivative in the THF-water system (Fig. 2). The aggregation of these molecules was indicated by the $10 \mathrm{~nm}$ red shift and the reduction of the maximum absorption in the UV spectrum. The maximum absorption peak gradually red shifts when increasing the self-assembly temperature, owing to the increasing aggregation of the nanostructure. The PSS solution was obtained after irradiating the THF solution with

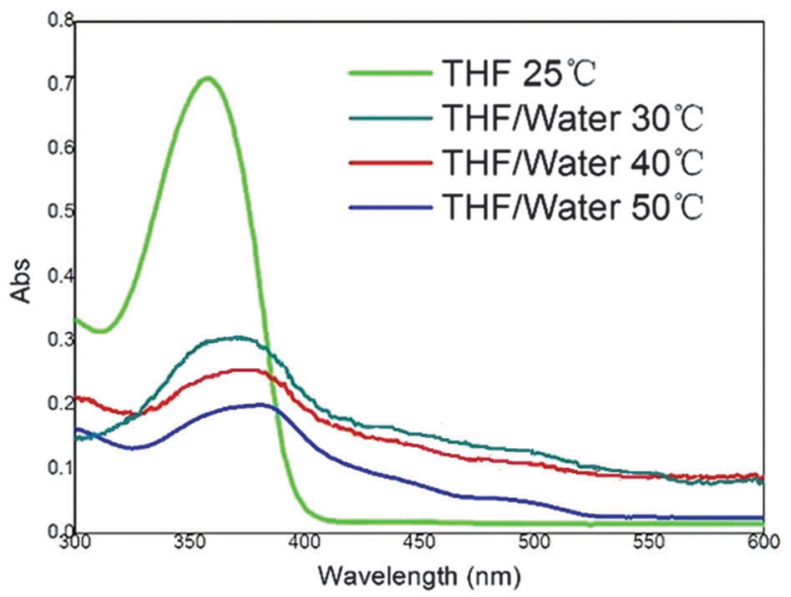

Fig. 2 Temperature-dependent UV-Vis spectra of diarylethene in THF-water systems.

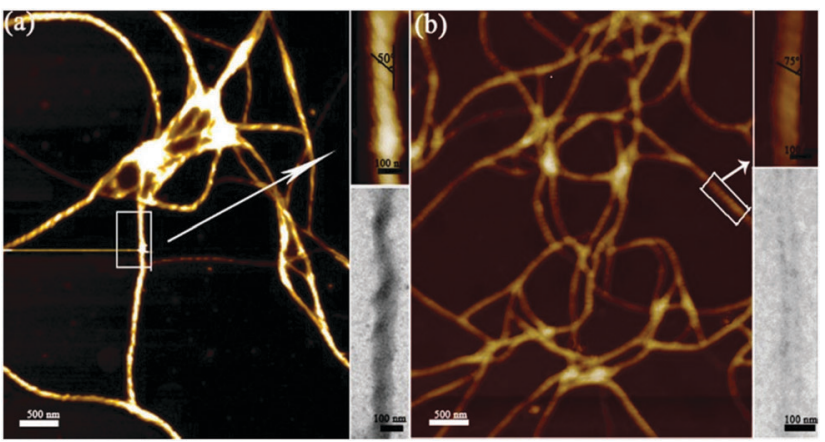

Fig. 3 The AFM image, enlarged AFM image (top right corner) and enlarged TEM image (bottom right corner) of the helical fibers and helical tubes formed from the diarylethene before (a) and after (b) UV irradiation.

$365 \mathrm{~nm}$ UV light for ten minutes: the PSS of this diarylethene also had similar self-assembly behaviour in the THF-water system by the same method. The closed ring isomer of the diarylethene is thermally unstable in solution because of the strong electron-withdrawing group ${ }^{17}$ but the formation of the dimers or multimers is very fast and the colored isomer is very stable in the aggregate state. From the atomic force microscopy (AFM) results, it is clearly observed that the nanostructure self-assembled by the PSS is more uniform and the helical pitch is more compact compared with the open-ring isomer (Fig. S2, ESI $\dagger$ ). The diameter of all nanostructures is about $90 \mathrm{~nm}$ and the pitch of the helix is about $42 \mathrm{~nm}$. Furthermore, the helical angle of the nanostructures changed from $50^{\circ} \pm 5^{\circ}$ to $75^{\circ} \pm 5^{\circ}$ after UV irradiation (Fig. 3). Transmission electron microscopy (TEM) observation confirmed that the as-assembled nanostructure from the open-ring isomer was a helical fiber structure, To our surprise, after UV irradiation a tubular nanostructure was observed as shown in Fig. 3 and Fig. S3 in the ESI; $†$ to the best of our knowledge, so far helical nanotube structures are very rare in self-assembly systems. ${ }^{18}$ In the aggregated state, the isomer is very stable and cannot be switched, so the nanofiber and the nanotube cannot be directly transformed by light.

Here we propose a possible mechanism to elucidate the formation of the helical nanofiber and the helical nanotube based on DFT calculations employing the hybrid B3LYP functional equipped with a $6-31 \mathrm{G}^{*}$ basis set. ${ }^{19}$ The monomeric form of the open-ring isomer has an energy $42 \mathrm{kcal} \mathrm{mol}^{-1}$ lower than the closed-ring structure in the gas phase, suggesting the former is more stable. Different dimeric conformations were tested (Table S1, ESI $\dagger$ ) and the most stable one has two intermolecular hydrogen bonds between the nitrogen of the $\mathrm{C} \equiv \mathrm{N}$ group and the malononitrile $\mathrm{C}-\mathrm{H}$ hydrogen of the neighboring monomer for both the open-ring and closed-ring isomers (Fig. S5, ESI $\dagger$ ). The difference of the two dimers is the orientation of the free malononitrile groups, which are on the same side of the plane defined by two hydrogen bonds for the closed-ring, or on different sides for the open-ring one. The tetramers were built based on the dimer structures. For the tetramer of the closed-ring isomer the $\mathrm{C}-\mathrm{F} \cdots \mathrm{H}-\mathrm{C}$ hydrogen bonds are formed between the dimers, whereas for the openring tetramer the $\mathrm{C} \equiv \mathrm{N} \cdots \mathrm{H}-\mathrm{C}$ hydrogen bonds are formed. 
Table 1 The optimized structures at B3LYP/STO-3G of the helical nanotube and helical nanofiber, closed-ring dimers (DP = 24, 72), open-ring dimers $(\mathrm{DP}=24)$

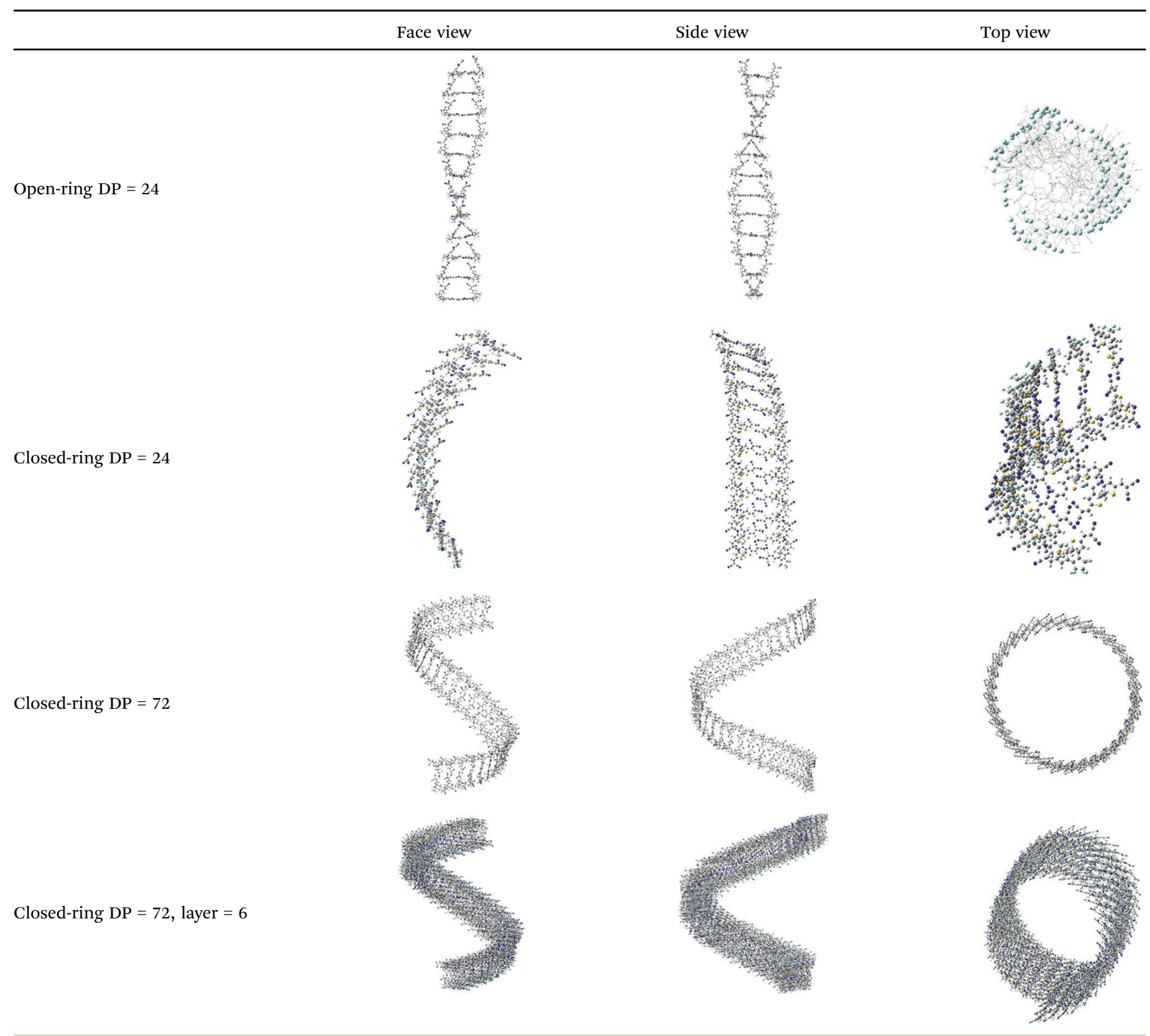

The formation of the dimer and tetramer lays the foundation for the nanostructure assembly. The optimized structures of 24 monomers at B3LYP/STO-3G are presented in Table 1. By extrapolation, the closed-ring assembly with the degree of polymerization (DP) of 72 is also shown. As can be seen, open-ring isomers assemble to form a nanofiber and closedring isomers form a nanotube. The small angle X-ray diffraction (SAXD) pattern of the helical nanofiber shows the molecules are arranged with a $1.88 \mathrm{~nm}$ interplanar spacing in the monolayer which is in accordance with the $1.8 \mathrm{~nm}$ interplanar spacing shown in Fig. S6 (ESI $\dagger$ ). The distances between the layers of the open-ring and the closed-ring assemblies are about $1.0 \mathrm{~nm}$ and $0.4 \mathrm{~nm}$ respectively, indicating that the open-ring polymer is organized more loosely. The experimental wall thickness of the nanotube is about $10 \mathrm{~nm}$, which is ten times bigger than the monolayer thickness, so we think that the helical nanotube is rolled from several monolayers. The rolling of the nanostructures is similar to the nanotubes which roll from a single bilayer as reported by Danino et al. ${ }^{20}$

In summary, we have constructed a helical nanostructure by self-assembly of a diarylethene derivative and successfully tuned the helical properties using light, as the molecular shape of the underlying monomer can be changed by ultraviolet or visible irradiation. When the diarylethene monomer changed from the open-ring isomer to the closed-ring isomer, not only does the helix angle of the self-assembly structure undergo a significant change but also the morphology of the aggregation state could be transformed from helical nanofiber to helical nanotube. The helical 
angle of the nanostructures could also be tuned by UV irradiation, which has great potential for smart soft materials and devices. The possible formation mechanism of the nanostructure was presented based on theoretical calculations.

We appreciate the support of "100 Talents" program of the Chinese Academy of Sciences, National Key Basic Research Program of China (Grant no. 2011CB935703). The authors thank Prof. Gang Chen, Chinese Academy of Sciences, for assistance with the SAXD analysis.

\section{Notes and references}

1 J. D. Watson and F. H. C. Crick, Nature, 1953, 171, 737-738.

2 (a) M. George and R. Weiss, Chem. Rev., 2006, 39, 489-497; (b) A. Miura, Z. Chen, H. Uji-i, S. D. Feyter, M. Zdanowska, P. Jonkheijm, A. P. H. J. Schenning, E. W. Meijer, F. Würthner and F. C. D. Schryver, J. Am. Chem. Soc., 2003, 125, 14968-14969; (c) I. Danila, R. F. Franc-ois, F. Piron, J. Puigmartí-Luis, J. D. Wallis, M. Linares, H. Ågren, D. Beljonne, D. B. Amabilino and N. Avarvar, J. Am. Chem. Soc., 2011, 133, 8344-8353; (d) P. Jonkheijm, P. V. D. Schoot, A. P. H. J. Schenning and E. W. Meijer, Science, 2006, 313, 80-83.

3 (a) J. V. Gestel, A. R. A. Palmans, B. Titulaer, J. A. J. M. Vekemans and E. W. Meijer, J. Am. Chem. Soc., 2005, 127, 5490-5494; (b) G. D. Pantos, P. Pengo and J. K. M. Sanders, Angew. Chem., Int. Ed., 2007, 46, 194-197; (c) G. L. Cui, H. Xu, W. Xu, G. C. Yuan, D. Q. Zhang, L. Jiang and D. B. Zhu, Chem. Commun., 2005, 277-278.

4 (a) T. Yamamoto, T. Fukushima, A. Kosaka, W. Jin, Y. Yamamoto, N. Ishii and T. Aida, Angew. Chem., Int. Ed., 2008, 120, 1696-1699; (b) J. P. Hill, W. Jin, A. Kosaka, T. Fukushima, H. Ichihara, T. Shimomura, K. Ito, T. Hashizume, N. Ishii and T. Aida, Science, 2004, 304, 1481-1483.
5 (a) A. P. H. J. Schenning, J. V. Herrikhuyzen, P. Jonkheijm, Z. Chen, F. Würthner and E. W. Meijer, J. Am. Chem. Soc., 2002, 124, 10252-10253; (b) A. Ajayaghost and K. V. Praveen, Acc. Chem. Res., 2007, 40, 644-656.

6 J. V. Gestel, A. A. Palmans, B. Titulaer, J. J. M. Vekemans and E. W. Meijer, J. Am. Chem. Soc., 2005, 127, 5490-5494.

7 L. Brunsveld, H. Zhang, M. Glasbeek, J. A. J. M. Vekemans and E. W. Meijer, J. Am. Chem. Soc., 2000, 122, 6175-6182.

8 (a) L. S. Li, H. Z. Jiang, B. W. Messmore, S. R. Bull and S. I. Stupp, Angew. Chem., Int. Ed., 2007, 46, 5873-5876; (b) H. Engelkamp, S. Middelbeek and R. J. M. Nolte, Science, 1999, 284, 785-788.

9 M. Ikeda, N. Tanifuji, H. Yamaguchi, M. Irie and K. Matsuda, Chem. Commun., 2007, 1355-1357.

10 K. Matsuda and M. Irie, Chem. Lett., 2000, 16-17.

11 U. Al-Atar, R. Fernandes, B. Johnsen, D. Baillie and N. Branda, J. Am. Chem. Soc., 2009, 131, 15966-15967.

12 A. Uyama, S. Yamazoe, S. Shigematsu, M. Morimoto, S. Yokojima, H. Mayama, Y. Kojima, S. Nakamura and K. Uchida, Langmuir, 2011, 27, 6395-6400.

13 S. Yagai, K. Ohta, M. Gushiken, K. Iwai, A. Asano, S. Seki, Y. Kikkawa, M. Morimoto, A. Kitamura and T. Karatsu, Chem. Eur. J., 2012, 18, 2244-2253.

14 S. Delbaere, J. Berthet, T. Shiozawa and Y. Yokoyama, J. Org. Chem., 2012, 77, 1853-1859.

15 S. L. Gilat, S. H. Kawai and J.-M. Lehn, J. Chem. Soc., Chem. Commun., 1993, 1439-1442.

16 J. Wang, H. Lu, R. Kamat, S. V. Pingali, V. S. Urban, J. J. Cheng and Y. Lin, J. Am. Chem. Soc., 2011, 133, 12906-12909.

17 M. Irie, Chem. Rev., 2000, 100, 1685-1716.

18 P. F. Duan, L. Qin, X. F. Zhu and M. H. Liu, Chem. - Eur. J., 2011, 17, 6389-6395.

19 C. T. Lee, W. T. Yang and R. G. Parr, Phys. Rev. B, 1988, 37, 785-789.

20 L. Ziserman, H. Y. Lee, S. R. Raghavan, M. Mor and D. Danino, J. Am. Chem. Soc., 2011, 133, 2511-2517. 